Pablo Herrera*

Javier García Fronti***

Universidad de Buenos Aires, Buenos Aires, Argentina.

Recibido: 14 de marzo de 2014

Concepto de evaluación: 16 de junio de 2014

Aprobado: 18 de junio de 2014

Artículo de investigación (C) 2014 Universidad Católica de Colombia. Facultad de Ciencias Económicas y Administrativas. Todos los derechos reservados

* Magíster en Gestión Económica y Financiera de Riesgos de la Facultad de Ciencias Económicas, Universidad de Buenos Aires. Investigador y docente del Centro de Métodos Cuantitativos Aplicados a la Economía y la Gestión (CMA), Universidad de Buenos Aires. Dirección de correspondencia: Córdoba 2122,

1120AAQ, Ciudad Autónoma de Buenos Aires, República Argentina. Correo electrónico: pabloherrera@economicas.uba.ar

** Doctor de la Universidad de Buenos Aires. Docente e investigador del Centro de Métodos Cuantitativos

Aplicados a la Economía y la Gestión (CMA), Universidad de Buenos Aires. Dirección de correspondencia: Córdoba 2122,

1120AAQ, Ciudad Autónoma de Buenos Aires, República

Argentina. Correo electrónico: javier.garciafronti@economicas.uba.ar

\section{Impacto del crédito gubernamental en el sistema financiero ${ }^{1}$}

\section{RESUMEN}

La crisis financiera mundial, que inició en el año 2008, impulsó un creciente interés en los temas relacionados con la estabilidad del sistema financiero y una consecuente necesidad de modificación en su regulación. Este trabajo propone un modelo teórico de tres agentes: intermediario financiero, firmas y gobierno, dentro del cual se demuestra que el otorgamiento de subsidios gubernamentales es más efectivo si se realiza a través de un intermediario financiero; lo que conlleva, a su vez, una mayor profundización financiera. La primera sección desarrolla los conceptos de prociclicidad, apalancamiento y riesgo sistémico. En la segunda sección se presenta el modelo y se realiza el ejercicio macroeconómico mencionado.

Palabras clave: sistema financiero, prociclicidad, apalancamiento, riesgo sistémico, créditos gubernamentales.

JEL: E32, G21, G28, G32

\section{Impact of the Governmental Credit on the Financial System}

\section{ABSTRACT}

The global financial crisis that began in 2008 triggered a growing interest in issues related to financial stability and a consequent need for change in their regulation. This work proposes a three-agents theoretical model financial intermediaries, firms and government- in which it is shown that the government subsidies granting is more effective if they are directed through a financial intermediary, carrying besides, to a further financial depth.

Este documento es resultado del proyecto de investigación UBACyT 2014-2017: Gobernanza macroprudencial sostenible: financiamiento de la innovación, del agro y sus impactos socioeconómicos. El caso de las pymes y los pequeños productores agropecuarios en Argentina, dirigido por la doctora María Teresa Casparri. Los autores agradecen las observaciones de los árbitros anónimos a la primera versión del documento. Cualquier error u omisión es responsabilidad de los autores. 
The first section develops the concepts of pro-cyclicality, leverage and systemic risk. The model is presented in the second section and the macroeconomic exercise is developed.

Keywords: Financial system, procyclicality, leverage, systemic risk, governmental credits.

\section{Impacto do crédito governamental no sistema financeiro}

\section{RESUMO}

A crise financeira mundial, que começou em 2008, impulsionou um crescente interesse nos temas relacionados com a estabilidade do sistema financeiro e uma consequente necessidade de modificação em sua regulação. Este trabalho propõe um modelo teórico de três agentes: intermediário financeiro, firmas e governos, dentro do qual se demonstra que a concessão de subsídios governamentais é mais efetivo se for realizado por meio de um intermediário financeiro; o que implica, ao mesmo tempo, um maior aprofundamento financeiro. A primeira seção desenvolve os conceitos de prociclicidade, alavancagem e risco sistêmico. Na segunda seção, apresenta-se o modelo e realiza-se o exercício macroeconômico mencionado.

Palavras-chave: sistema financeiro, prociclicidade, alavancagem, risco sistêmico, créditos governamentais. 


\section{INTRODUCCIÓN}

La crisis financiera mundial que inició en el año 2008 impulsó un creciente interés en los temas relacionados con la estabilidad del sistema financiero y una consecuente necesidad de modificación en su regulación. Su relevancia se acentúa al comprender el fuerte impacto en la economía real de los problemas en el sector financiero. Los lados real y financiero de la economía se retroalimentan mutuamente exacerbando la expansión del ciclo económico y haciendo mucho más severa su contracción. En este sentido, el sistema bancario es procíclico. Por esta razón, una política monetaria que persiga un crecimiento sostenido y estable del producto debe tener en cuenta esta característica a la hora de proponer regulaciones. En particular, los bancos utilizan un apalancamiento (cociente de los activos sobre el capital propio) que por naturaleza es procíclico. Las entidades financieras tienen el incentivo de modificar la composición de su deuda de acuerdo con la instancia del ciclo económico, con la que provocan que el sistema financiero sea especialmente propenso a la gestación de un riesgo de carácter sistémico.

Este trabajo, más allá de evaluar la relación entre el tamaño del sistema financiero y el crecimiento del producto (Beck, Degryse \& Kneer, 2014; Chen \&Imam, 2014), propone un modelo teórico simple que permite entender la interacción y la articulación entre el sector público y el sector financiero. Sobre este, se realiza el ejercicio de asumir un shock exógeno dado por un programa gubernamental de créditos. Formalmente, luego de plantear un modelo de dos agentes - intermediario financiero y firmas-, se incorpora un tercer agente, el Gobierno, y se demuestra que el otorgamiento de subsidios gubernamentales es más efectivo si se realiza a través de un intermediario financiero.

Lo anterior radica en el mecanismo de apalancamiento implementado por el banco tipo. Sin embargo, además de un mayor aumento en el nivel de producto, el aumento del capital bancario genera consecuencias en lo que respecta a la profundización financiera. En el proceso de reacomodación en la estructura del balance bancario, en pos de un aumento de los beneficios del intermediario financiero, se terminan profundizando las interconexiones dentro del sistema financiero y se gesta así un potencial riesgo sistémico que en el caso de materializarse anula por completo los objetivos perseguidos por la implementación de la política gubernamental de otorgamiento de subsidios.

Para lograr el objetivo propuesto, la primera sección desarrolla los conceptos de prociclicidad, apalancamiento y riesgo sistémico. El primero está directamente ligado a la evolución del ciclo real, haciendo que las fluctuaciones del ciclo financiero sean más pronunciadas que las fluctuaciones del producto. El apalancamiento se define a partir de la hoja de balance del sistema financiero y se enfatiza en su carácter procíclico. Por último, se define el riesgo sistémico a partir de una descripción de fenómenos que lo caracterizan. Se indaga sobre la variación en la composición de los pasivos de las hojas de balance del sector financiero en diferentes etapas del ciclo económico. En la segunda sección se presenta el modelo y se realiza el ejercicio macroeconómico mencionado.

\section{EL SISTEMA FINANCIERO}

El sistema financiero es una parte fundamental de la economía. Su principal función es la canalización de los ahorros hacia la inversión, lo que a su vez estimula el crecimiento económico. Por esta misma razón, la estabilidad del sistema financiero es imprescindible para la prosperidad económica. A raíz de la última crisis financiera mundial, que se desató hacia finales de la primera década del 2000, la elaboración de trabajos referidos a las regulaciones financieras se encuentra en pleno auge.

La restricción presupuestaria del sistema financiero, dada por el balance de este sector, sintéticamente se puede describir de la siguiente manera. En el lado de los activos, se encuentran los préstamos realizados a terceros; mientras que en el lado de los pasivos, se encuentran los aportes realizados por los accionistas del sector financiero; 
Figura 1.

Esquema del negocio financiero

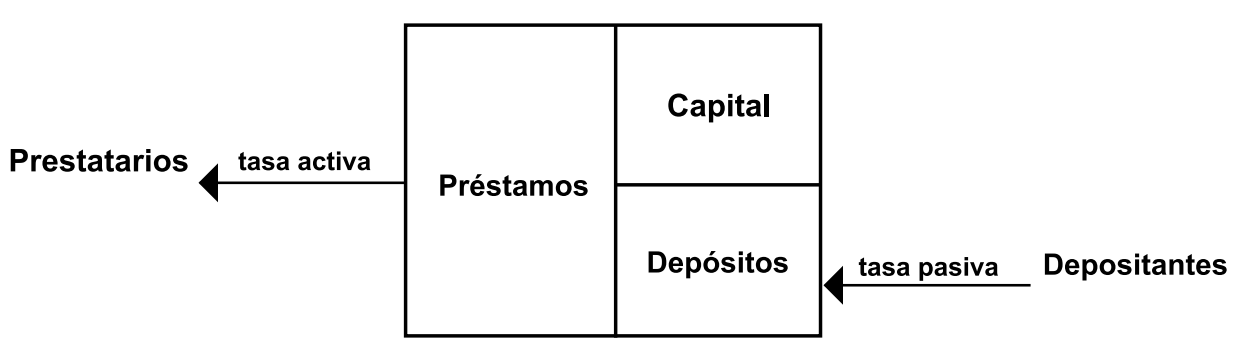

Fuente: elaboración de los autores.

lo que generalmente se conoce como capital bancario, y las deudas de la entidad para con terceros. Esta deuda está dada por los depósitos que realizan los terceros en una entidad en particular. El negocio financiero consiste en captar depósitos, por los que se paga una tasa de interés (pasiva) que es menor a otra tasa de interés (activa) a la cual se otorgan préstamos (figura 1).

Los modelos representativos del sistema financiero generalmente proponen como restricción presupuestaria un balance representativo del sector. Estos modelos se diferencian entre sí a partir de la composición que proponen sobre los balances financieros. Como referentes de esta manera de desarrollar modelos teóricos se pueden citar los siguientes trabajos: Anastasi y Balzarotti (2012), donde se distinguen diferentes tipos de activos; Hahm, Shin y Shin (2012) y Bruno y Shin (2013), donde se distinguen diferentes tipos de pasivos; Céspedes, Chang y Velasco (2012), donde distinguen entre diferentes tipos de capital propio.

Si bien la estabilidad del sistema financiero es indispensable para el buen desempeño económico en cualquier parte del mundo, es preciso saber diferenciar cómo se compone dicho sector en cada país. Como asevera Mishkin (2007), el sector bancario, con diferente peso entre las distintas economías, es el principal canalizador de los ahorros hacia la inversión productiva. Por esta razón, a lo largo del desarrollo de este artículo no se realizará ninguna distinción entre el sector financiero y el sector bancario. ${ }^{2}$ Sin embargo, hay ciertas características del sector financiero que son independientes de la economía en cuestión, y tres de ellas se analizan a continuación: la prociclicidad, el apalancamiento y el riesgo sistémico.

Con el fin de recorrer diversos aspectos del sistema financiero, esta sección se estructura de la siguiente manera: en el primer apartado, se explica el concepto de prociclicidad y las tres fuentes que generan que el negocio bancario sea procíclico. La primera de estas fuentes está referida a aspectos propios de la actividad financiera; la segunda, a las fallas de mercado existentes dentro del sector, y la última, a las propias regulaciones financieras.

En el siguiente apartado, se define el concepto de apalancamiento bancario y se explica por qué presenta la característica de ser procíclico. Posteriormente, mediante un ejemplo sencillo, se muestra que, a pesar de los intentos regulatorios de exigir un apalancamiento constante,

2 A grandes rasgos, se puede hacer una distinción entre intermediarios financieros bancarios e intermediarios financieros no bancarios. Entre los primeros se encuentran el Banco Central que regula el sistema financiero, la banca privada y la banca pública, las cajas de ahorro y las cooperativas de crédito. Entre los segundos se encuentran las compañías aseguradoras, los fondos de pensiones o mutualidades, las sociedades de inversión inmobiliaria, los fondos de inversión, las sociedades de crédito hipotecario, las entidades de leasing, las de factoring, las sociedades mediadoras del mercado de dinero y las sociedades de garantía recíproca. Mientras los primeros se caracterizan porque algunos de sus pasivos son pasivos monetarios aceptados generalmente por el público como medios de pago, los segundos se caracterizan porque sus pasivos no son dinero. 
la reacomodación de los pasivos que realizan las entidades financieras en la etapa de crecimiento económico hace que el sector sea altamente inestable; esta inestabilidad se ve aún más pronunciada con un apalancamiento procíclico. En el último apartado, a partir de la definición de prociclicidad y de apalancamiento, se define el concepto de riesgo sistémico.

\section{Prociclicidad}

Una característica del sistema financiero es su prociclicidad. ${ }^{3}$ Esto implica que las fluctuaciones de este sector, si bien tienen el mismo sentido que el ciclo de la economía real, son mucho más volátiles. Debido a que ambos lados se retroalimentan mutuamente, la prociclicidad del sector financiero exacerba la expansión del ciclo económico y también hace mucho más severa su contracción. Por esta razón, para obtener como resultado un crecimiento sostenido y estable del producto, lo que constituye el objetivo último de cualquier tipo de política macroeconómica, es necesario que se estabilice también el sistema financiero, siendo su prociclicidad un factor clave. Como se menciona en Gual (2009), existen tres fuentes de prociclicidad en el sistema bancario. La primera está referida a ciertos aspectos propios de la actividad financiera; la segunda, a las fallas de mercado existentes dentro del sector, y la última, a las propias regulaciones financieras.

En cuanto a la primera fuente de prociclicidad, el autor la vincula directamente con la competencia por una mayor cuota de mercado. Durante un periodo de expansión económica, el costo de otorgamiento de préstamos es menor. En este periodo, la expansión del crédito y la competencia por ganar una mayor cuota de mercado son mayores. Durante la fase expansiva del ciclo económico, un mayor número de entidades bancarias disponen de mayores fondos para llevar a cabo estrategias de captación de cuotas de mercado y, por lo tanto, las

$3 \quad$ Hamann, Hernández, Silva y Tenjo (2013) realizan un estudio empírico en el cual evidencian la prociclicidad del sistema financiero colombiano en el periodo 1994-2012. rebajas en los costos de los préstamos otorgados tenderán a ser más importantes. En los periodos de contracción, solo las entidades con un mayor capital pueden financiar las pérdidas a corto plazo y la competencia en precios tiende a ser menor.

La segunda fuente de prociclicidad, referida a las fallas de mercado dentro del sistema financiero, encuentra su origen en la información asimétrica entre los participantes de este negocio y en las externalidades que lo afectan. Los participantes del negocio financiero se clasificarán de la siguiente manera: depositantes, accionistas, gestores/prestamistas y prestatarios. Entre todos ellos hay recurrentes problemas de información asimétrica que contribuyen con la explicación de la prociclicidad del sistema financiero. La posición que presenta cada uno de estos agentes dentro de una estandarización básica del balance bancario se da a conocer en la figura 2 .

Figura 2.

Participantes del negocio financiero

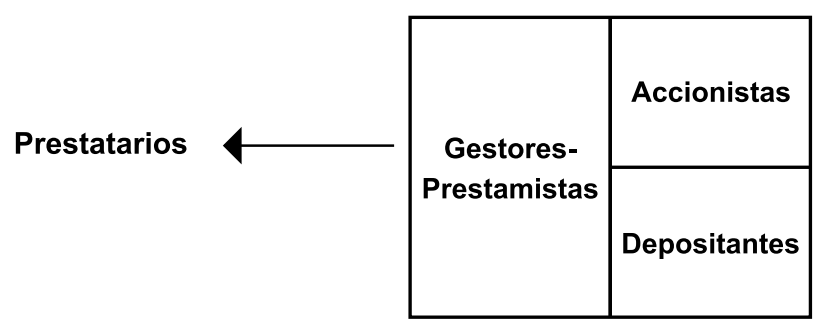

Fuente: elaboración de los autores.

La información asimétrica que se produce entre los prestamistas y los prestatarios radica en el hecho de que los primeros desconocen el uso que los segundos darán a los fondos otorgados por la entidad financiera. Este problema es el de riesgo moral. Para disminuir los potenciales problemas que conlleva este tipo de información asimétrica, los prestamistas suelen exigir a los prestatarios una garantía que se vincula directamente con el valor de algún activo real. ${ }^{4}$ Así, la oferta de crédito acaba

$4 \quad$ Una excelente referencia para poder modelizar los problemas de información asimétrica que existen dentro del sistema financiero se puede encontrar en Bebczuk (2003). 
dependiendo del valor de estos activos, que suele ser altamente procíclico. La posibilidad de los prestatarios de acceder a algún tipo de financiamiento depende también de su situación financiera; la cual, reflejada en los flujos de caja y la posición del balance, se refuerza en la etapa expansiva del ciclo económico, y consecuentemente la obtención de préstamos es más accesible. ${ }^{5}$

Otro problema de información asimétrica se produce entre los propietarios del pasivo de las entidades financieras. Esto es, entre los depositantes y los accionistas. Los depositantes son agentes que, en comparación a los accionistas, están interesados en adoptar un menor riesgo. A su vez, los depositantes tienen poca influencia sobre las posiciones que toman los gestores. La manera que tienen los depositantes de controlar el tipo de inversión por parte de los gestores es a través de la alineación de los incentivos de los capitalistas. Esto suele realizarse mediante requisitos de capitales mínimos que exigen los reguladores financieros, lo que pone en riesgo un mayor capital de los accionistas en cada inversión realizada por los gestores. Así, la participación de la deuda sobre el capital bancario suele comportarse de manera procíclica. En épocas de expansión económica, las entidades financieras, al igual que los prestatarios, mejoran sus flujos de caja y sus posiciones de balance. En consecuencia, pueden acceder a un mayor nivel de depósitos, lo que se traduce en una mayor participación de deuda en el pasivo del sector.

Entre los accionistas y los gestores del sistema financiero también se produce un tipo de información asimétrica; surge el clásico problema de principal-agente. Mientras que los accionistas (principal) están interesados más por los beneficios a largo plazo que se puedan obtener dentro

$5 \quad$ Hay que resaltar que hasta el momento solo se ha hecho referencia a los problemas de riesgo moral que existen dentro del sistema financiero. Sin embargo, es necesario aclarar que dentro de este sector también existen problemas de información relacionados con la selección adversa en el proceso de demanda de crédito. Esto agrega una explicación adicional a la existencia de prociclicidad en el sistema financiero ya que los estándares de crédito (créditos con garantía) se relajan durante los periodos expansivos y se endurecen en los periodos recesivos. del sector, los gestores (agente) asumen grandes riesgos para maximizar los beneficios a corto plazo. Así, los gestores financieros en pos de maximizar los beneficios a corto plazo, en etapas de expansión económica, pueden asumir mayores riesgos otorgando una mayor cantidad de préstamos, lo que acentúa la prociclicidad del sistema financiero.

Junto con los problemas presentados de información asimétrica, las externalidades negativas constituyen una fuente que acentúa las fallas del mercado financiero. Las externalidades negativas dentro de este sector serán comprendidas como situaciones en las que el accionar de una entidad financiera individual, en busca de un beneficio propio, incide negativamente en el resto de las entidades de este tipo. A raíz de este tipo de externalidades, el mercado financiero queda en una situación de desequilibrio.

Gual (2009), como ejemplo de externalidad negativa, explica los pánicos bancarios. La esencia del pánico bancario radica en el siguiente fenómeno: ante la percepción por parte de un grupo reducido de depositantes de la falta de liquidez de un banco, se puede producir la retirada masiva de depósitos del sistema bancario. El grupo de depositantes es disperso y, en general, cada uno cuenta con información imperfecta en cuanto al estado de los bancos. Así, un problema de liquidez de una entidad financiera individual se puede traducir en un problema de solvencia del sector financiero en su conjunto. Los pánicos bancarios suelen producirse durante recesiones y son amplificadores de la contracción económica consecuente.

Otro tipo de externalidad negativa se puede producir por la acumulación preventiva de capital. Como se ha explicado anteriormente, durante épocas recesivas, los accionistas intentan aumentar la capitalización bancaria. En esta etapa del ciclo económico, donde los capitales son escasos, la excesiva capitalización por parte de una entidad financiera en particular puede llevar a la inestabilidad del sistema en su conjunto.

Para aumentar la capitalización, las entidades tienen dos posibilidades. La primera implica la reducción de préstamos otorgados por la entidad; la segunda radica en la venta de activos. 
Apalancamiento producido por un aumento en el valor de los activos

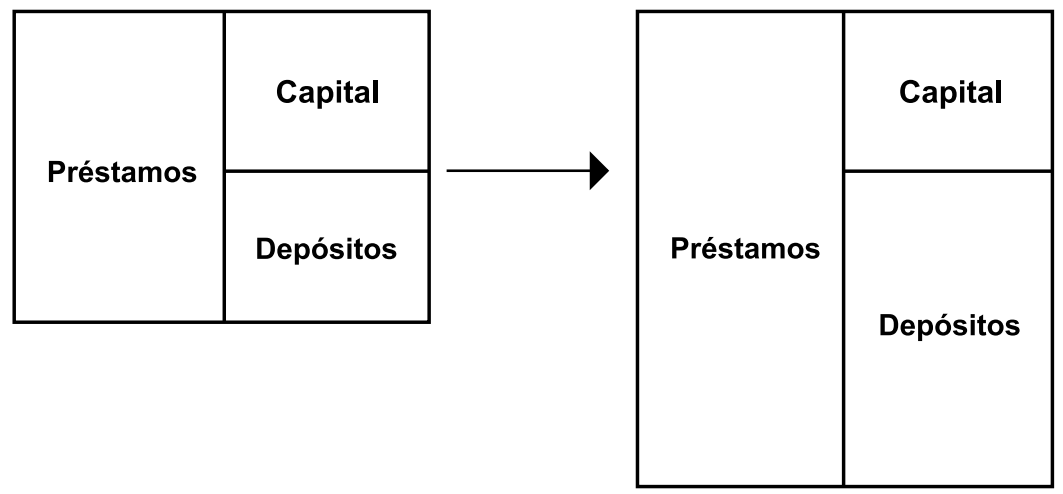

Fuente: elaboración de los autores con base en Adrian y Shin (2013).

Ambas posibilidades hacen que la acción individual de la entidad derive en un problema del sector financiero agregado, ya sea por la reducción de créditos otorgados o por la reducción del valor de los activos vendidos. Consecuentemente, se produce un problema de solvencia dentro del sector. Un aspecto clave para entender el carácter procíclico del sistema es el nivel de apalancamiento de sus agentes, este tema se analiza en el próximo apartado.

\section{Apalancamiento}

Las variaciones en las hojas de balance de las entidades financieras se encuentran estrechamente vinculadas con las que experimenta el mercado. Esto es, un cambio en el precio de los activos reales se refleja instantáneamente en la hoja de balance del sistema financiero. Como se explicó previamente, durante la época de auge económico, se incrementa la oferta de préstamos bancarios. En esta fase, a la vez que se registran elevados beneficios bancarios, los riesgos cuantificables son bajos (primera fuente de prociclicidad). De esta manera, el crecimiento en la oferta de préstamos, sumado a una percepción de ratios de capital (inversa del nivel de apalancamiento) reforzados, da como resultado una merma en los requerimientos de capital mínimo solicitados por los depositantes (a través de las regulaciones). La consecuencia inmediata es un mayor apalancamiento del sistema financiero. El riesgo que conlleva este proceso se hace evidente en las etapas contractivas del ciclo económico (figura 3).

Debido al carácter procíclico que muestra el apalancamiento, es comprensible que una de las bases de la regulación financiera sea solicitar ratios mínimos de capital. El objetivo de esta medida es poder mantener constante el apalancamiento de las entidades financieras. Para mostrar las consecuencias de un apalancamiento constante, siguiendo a Adrian y Shin (2013), se realizará un pequeño ejercicio a modo de ejemplo. En el desarrollo de este ejercicio se considera un banco en el cual todos los préstamos que otorga son asegurados en la bolsa comercial. En consecuencia, el valor de los activos varía con el precio general de la economía. ${ }^{6}$

6 Esta es una característica de economías con un mercado financiero desarrollado. Sin embargo, el ejemplo a desarrollar también aplica para economías con sectores financieros poco profundos, ya que estos, entre sus activos, conservan diferentes tipos de bonos (estatales y privados) cuyos precios varían también con el precio general de la economía. 
Supóngase la hoja de balance de una entidad financiera, la cual tiene que mantener un apalancamiento constante. Esta entidad en su activo ofrece préstamos por un valor de 100, los cuales son compensados en el pasivo con deuda por un valor de $90 \mathrm{y}$ capital propio por un valor de 10. Ahora, supóngase que el precio general de la economía aumenta en un $10 \%$. Consecuentemente, el valor de los préstamos pasa a valer 101. Por el lado de los pasivos, este aumento es respaldado en su totalidad por un aumento del capital propio que pasa a valer 11 ya que el valor de los títulos accionarios que se conservan también crece en una unidad. En este caso, el valor del apalancamiento disminuiría y, por consiguiente, necesitaría endeudarse 9 unidades adicionales para aumentar el valor de sus activos hasta 110, y así conseguir el objetivo de apalancamiento constante.

Este mismo mecanismo también funciona de manera inversa. Partiendo de la situación final planteada recientemente, el balance de la entidad financiera está compuesto por 11 unidades de capital propio y 99 de deuda. Así, el valor de los préstamos es de 110. Ahora, el precio de los títulos baja en una unidad. El valor del capital propio pasará a ser de 10 y los préstamos otorgados pasarían a valer 109. En este nuevo escenario, el apalancamiento de la entidad financiera está por encima del objetivo de 10. En consecuencia, lo que se hará es reducir el valor de la deuda a partir de la venta de títulos hasta llegar al apalancamiento objetivo. En respuesta a un incremento del valor de los activos de la hoja de balance de una institución financiera, el apalancamiento de esta institución en particular en un primer momento disminuye, y mantiene un mayor nivel de capital. Posteriormente, esa entidad puede captar un mayor nivel de deuda hasta llegar al nivel de apalancamiento originalmente establecido; sin embargo, este no es el final de la historia.

El mayor nivel de capital que obtienen las entidades financieras, en un primer momento, es utilizado en pos de aumentar aún más su hoja de balance, tomando una mayor deuda. El punto de la cuestión es el tipo de deuda que adquieren estas entidades, que se caracteriza por ser una deuda a corto plazo, generalmente proveniente de otras entidades financieras. A su vez, en el lado de los activos, buscan potenciales tomadores de deuda a los que ellos les puedan prestar, generalmente prestatarios financieros. Así, cuando el precio de los títulos aumenta, el ajuste hacia arriba del apalancamiento lleva a la compra de más títulos. Consecuentemente, el ajuste en el apalancamiento y los cambios en los precios se refuerzan mutuamente uno con otro, lo que amplifica el ciclo financiero. La reacción ante los cambios de precios es aún más fuerte cuando el apalancamiento de las entidades financieras es procíclico.

Definida la liquidez de mercado como la tasa de crecimiento de la hoja de balance de las entidades financieras, esta queda íntimamente relacionada con la manera como las entidades financieras se endeudan y buscan tomadores de créditos. Si la liquidez del mercado financiero no es lo suficientemente perfecta, un aumento de la demanda de títulos puede impulsar hacia arriba aún más los precios. De esta manera, el efecto de retroalimentación queda más reforzado; lo que hace que un aumento en el valor de la hoja de balance demande más títulos y, por consiguiente, aumente por presión de la demanda el precio de estos últimos, lo que vuelve a aumentar el valor de la hoja de balance. Este mismo mecanismo funciona también en sentido inverso.

Si ante el aumento del precio de activos reales, los intermediarios financieros no acrecentaran su nivel de deuda a corto plazo, el efecto perjudicial de la prociclicidad no se produciría. Sin embargo, este no es el caso. Las entidades financieras, ante un cambio en la cuantía de la hoja de balance, modifican la composición de su deuda, disminuyendo la proporción de pasivos a largo plazo. El resultado final es un descalce de plazos entre los activos y los pasivos que mantiene la entidad. Este resultado se ve agravado por la naturaleza del negocio bancario que se explicó al principio del presente apartado, lo que conduce a una correlación positiva ente las variaciones en el valor de la hoja de balance y el ratio de apalancamiento.

En resumen, el apalancamiento de carácter procíclico provoca que la intermediación financiera sea altamente inestable. Por eso, es importante 
analizar al riesgo sistémico en términos de la prociclicidad y el apalancamiento, siguiendo a De Bandt y Hartmann (2000); en el siguiente apartado se hace una exposición de lo que se entiende por riesgo sistémico.

\section{Riesgo sistémico}

El riesgo sistémico es otra de las características inherentes al sistema financiero. A su vez, una crisis sistémica dentro de este sector puede generar consecuencias adversas en el lado real de la economía. Como se mencionó previamente, el sector financiero funciona como canalizador de ahorros hacia inversiones reales. El buen funcionamiento de este sector consiste en poder hacer eficiente esa canalización para así obtener altos retornos de las inversiones realizadas. Una crisis sistémica se puede definir como un evento sistémico que afecta a un número considerable de instituciones financieras o de mercados. Esto se produce a partir de una falla en el funcionamiento del negocio financiero.

El riesgo sistémico puede ser definido como el riesgo de experimentar eventos sistémicos. Esto es, un evento que tenga consecuencias de segunda vuelta sobre una institución financiera individual, o un evento que afecte a más de una institución. El carácter geográfico de este tipo de riesgo puede ser regional, nacional o, incluso, internacional. Para la definición de este concepto, es útil distinguir entre tres momentos de un evento de carácter sistémico. El primer momento es el shock; el segundo, el mecanismo de transmisión, y el tercero, el impacto.

Un shock puede ser idiosincrático o sistemático. El primero es aquel que, inicialmente, afecta el buen funcionamiento de una entidad financiera individual; el segundo, en cambio, afecta a un conjunto de entidades al mismo tiempo. Un ejemplo de shock idiosincrático podría estar dado por la quiebra de una entidad financiera individual debido al default de los prestatarios de esa entidad. Un ejemplo de shock sistemático podría estar dado por las fluctuaciones del producto de una economía, o las variaciones de la tasa de interés, que afectan a las entidades en su conjunto. Mientras, el primer tipo de shock permite la cobertura a partir de una diversificación, el segundo no.

El mecanismo de transmisión se puede dar en dos niveles. El primero se produce de una entidad financiera individual al resto de las entidades dentro de este sector. El segundo se produce desde el lado financiero al lado real de la economía. Cada uno de estos niveles de los mecanismos de transmisión trae severas consecuencias. El primer nivel provoca fallas en el negocio financiero que se van acumulando, y se traduce al segundo nivel, lo que provoca saltos discretos en los niveles de precios de la economía.

El impacto de evento sistémico se puede diferenciar en dos niveles. El primer nivel es el horizontal, el cual hace referencia a un impacto del evento sistémico sobre el sistema financiero en su conjunto. El segundo nivel es el vertical, que se produce cuando el evento sistémico, generado dentro del sistema financiero, tiene un impacto sobre el producto de la economía real.

Una vez detallados estos tres momentos de los eventos sistémicos, se procede a describir una serie de características del sistema financiero, las cuales hacen que este sector sea vulnerable a la gestación del riesgo sistémico. La primera hace referencia a los problemas de información inherentes dentro del sector; la segunda, a la estructura bancaria, y la tercera, a las interconexiones existentes entre las distintas entidades financieras. Estas tres características se vinculan directamente con los conceptos de prociclicidad y apalancamiento previamente analizados.

En cuanto a los problemas de información, como se explicó previamente, estos se producen entre los diferentes agentes que intervienen en el mercado financiero. El negocio financiero permite trasladar intertemporalmente el poder de compra de los agentes. Esto se realiza a partir de las expectativas sobre los precios futuros de los activos que mantienen en su cartera. Cuando la incertidumbre crece debido a un aumento en los problemas de información, se pierde confianza en el mercado financiero. Consecuentemente, las variaciones en los niveles de precios producidas por las decisiones de consumo e inversión pueden ser agudas. 
En relación con la estructura bancaria, es importante remarcar el hecho de que la integridad de una entidad bancaria no se define únicamente por la rentabilidad que presenta. El negocio financiero consiste en captar depósitos, por los que se paga una tasa de interés (pasiva) que es menor a otra tasa de interés (activa), a la cual se otorgan préstamos. Sin embargo, por más que esta entidad sea rentable, para que la misma sea considerada como segura es necesario mantener una cierta calidad en el nivel de su deuda (a mayor plazo y menor tasa de interés, mejor calidad de deuda) para asegurar la confianza de los depositantes. Ante una mala percepción de los depositantes acerca de la integridad de la entidad, se puede producir rápidamente una corrida bancaria.

Con respecto a las interconexiones bancarias, es un hecho que la red que comunica a las entidades financieras se profundiza a medida que la economía se expande. A mayor nivel de profundización financiera, la falla de un banco individual afecta inmediatamente la capacidad de pago de los demás intermediarios financieros, lo cual aumenta la probabilidad de ocurrencia de una crisis sistémica, en principio, de impacto horizontal. Una situación de crisis más profunda, con impacto vertical, puede llegar a afectar el crecimiento económico, fenómeno que genera la profundización de las interconexiones bancarias.

Dentro de la deuda de la entidad para con terceros -es decir, los depósitos bancarios-, se puede realizar una segmentación entre diferentes tipos de pasivos. ${ }^{7}$ A grandes rasgos, se puede hacer una distinción entre pasivos principales y pasivos no principales. El límite de esta distinción radica en el plazo de los depósitos y la tasa que se paga por estos. Un pasivo principal se caracteriza por ser de largo plazo y pagar tasas bajas si se compara con un pasivo no principal, que adicionalmente es un depósito de menor plazo. Los pasivos principales que

$7 \quad$ Hahm et al. (2012) realizan una descomposición de los pasivos entre core y non-core. Estos últimos son los que provienen de otras entidades financieras $y$, consecuentemente, son los más inestables y sensibles a las fluctuaciones de precios. En el trabajo, se explica claramente la correlación que existe entre este tipo de pasivos y la evolución del riesgo sistémico que se gesta en el sector financiero. mantienen los bancos son los pasivos cuyos acreedores son los ahorristas domésticos. Los depósitos minoristas son el mejor ejemplo de estos pasivos principales. En el otro extremo, las obligaciones de un intermediario financiero para con otro intermediario financiero pasarían a formar parte de los pasivos no principales con los que cuenta un banco.

El excesivo crecimiento de los activos de las entidades financieras se refleja en el lado de los pasivos por un cambio en la composición de los fondos bancarios. Los pasivos principales que mantienen los bancos crecen en línea con el crecimiento de la riqueza total de los hogares. En un boom de créditos, cuando estos crecen muy rápido, el agregado de los depósitos minoristas no es suficiente para respaldar el incremento del crédito bancario $y$, de esta manera, es necesario explotar otras fuentes de financiamiento como son otros intermediarios financieros. Así se altera la composición de los depósitos bancarios, reduciendo la proporción de pasivos principales-no principales. ${ }^{8}$

El crecimiento de los pasivos no principales acompaña a la reducción de la madurez de los depósitos bancarios. Es decir, los pasivos de corto plazo dentro de una entidad financiera surgen como consecuencia de largas cadenas de intermediación dentro del mismo sector. El alargamiento de estas cadenas aumenta las interconexiones entre las diferentes entidades financieras. La prevalencia de mayores deudas a corto plazo es una consecuencia natural del incremento de los pasivos no principales.

Un crecimiento excesivo de activos y una mayor dependencia de pasivos más riesgosos están estrechamente ligados al aumento del riesgo sistémico y a la interconexión entre las entidades financieras. En un auge, cuando el crédito crece rápidamente, el balance de los bancos supera la financiación básica disponible, y el crecimiento de los activos se termina reflejando en una gran exposición interbancaria.

8 En Hamann et al. (2013) se evidencia no solo que el sistema financiero colombiano es procíclico, sino también que el ratio de pasivos no principales/principales es un buen indicador de la etapa del ciclo económico. 
Con la explicación realizada, se puede evidenciar una estrecha conexión entre la prociclicidad, la interconexión y el stock de pasivos no principales que mantiene el sistema bancario. En un auge, se da la conjunción de tres factores: el rápido incremento de préstamos otorgados, la mayor proporción de pasivos no principales sobre el total de pasivos y el aumento del riesgo sistémico a través de los préstamos cruzados entre intermediarios financieros. Así, el riesgo sistémico es procíclico y el excesivo crecimiento de activos descansa en el incremento de las interconexiones bancarias.

En la siguiente sección, se desarrolla un modelo teórico matemático para poner de manifiesto los conceptos desarrollados hasta el momento. Dentro de este modelo, se ensayará un shock exógeno dado por una política gubernamental en particular, a saber, un programa de créditos. Esto permitirá dilucidar los beneficios y los perjuicios que la intermediación financiera provoca sobre la economía real. Así, el armado de este modelo es esencial para poder corroborar la hipótesis planteada dentro de este artículo: el armado de un modelo teórico-matemático que represente adecuadamente la naturaleza de la intermediación financiera es una herramienta útil para dilucidar los beneficios y los perjuicios de los programas de créditos gubernamentales dirigidos al sector financiero.

\section{EL MODELO}

Luego de la última crisis financiera mundial, la elaboración de modelos representativos del sistema financiero, o de alguna de sus características, cobró especial relevancia. Estos modelos son de diferente naturaleza e intentan explicar diversos fenómenos de este sector en particular. Los más generales, a partir de una descripción del sistema financiero, intentan incorporar la mayor cantidad de características posibles. Muchos hacen referencia a la vinculación existente entre el lado real y el lado financiero de la economía. En esta misma línea, hay modelos que intentan captar los efectos de las regulaciones financieras vigentes para argumentar a su favor o en contra.
Otros modelos hacen énfasis en economías particulares, y en el caso de ser abiertas, incluyen la variable tipo de cambio e identifican su incidencia dentro del sector financiero. A continuación, se presenta una breve descripción de una serie de trabajos que incorporan características relevantes para la confección del modelo propuesto en este artículo. Cabe destacar que no se pretende realizar una revisión exhaustiva de los modelos teóricos que representan el sistema financiero, sino brindar un aval y un sustento teórico al modelo que se presenta en el siguiente apartado.

En Goodhart, Sunirand y Tsomocos (2006), se definen ciertas características que debería incluir un modelo teórico para poder representar correctamente el comportamiento del sistema financiero de una economía. Entre estas se incluyen: la heterogeneidad de agentes que representen el sistema financiero y de agentes que representen el lado real; la inclusión de probabilidades de default sobre préstamos otorgados; la introducción de un mercado monetario, y, adicionalmente, la posibilidad de realizar mediciones empíricas a partir del modelo. Como se verá más adelante, la heterogeneidad de agentes se puede conseguir a partir de la distinción entre los préstamos otorgados y los depósitos captados por una entidad financiera modelo. A su vez, las probabilidades de default pueden incluirse a través de diferentes restricciones que se imponen sobre el funcionamiento de la entidad.

En Borio (2012) se describen tres características del ciclo financiero que también deberían ser incluidas dentro de un modelo. En primer lugar, hace referencia a la causalidad que se da desde la etapa ascendente hacia la etapa descendente del ciclo. En segundo lugar, destaca la importancia de incluir dentro del modelo la distinción entre los stock de capital y de deuda, remarcando que esas variables sean incorporadas de manera endógena y no exógena. En tercer lugar, le concede importancia a la distinción entre producto potencial como no inflacionario y como producto sustentable. Como se argumentó en la primera sección, la distinción entre el stock de capital y de deuda y su generación endógena dentro del modelo permitirían explicar 
la evolución del riesgo sistémico dentro del sistema financiero.

Yendo más allá de las características generales que debería incluir un modelo de carácter financiero, Marcheggiano, Miles y Yang (2013) y Powell, Miller y Maier (2011) son ejemplos de una aplicación práctica de estos modelos, de carácter teórico-matemático, que permiten analizar el capital bancario óptimo incluyendo probabilidades de default. Al mismo tiempo, posibilitan abarcar la discusión acerca de los requisitos mínimos de capital exigidos por las recomendaciones de Basilea, y su posible consecuencia sobre el funcionamiento del sistema financiero, y ponen de manifiesto el papel fundamental que cumple el capital bancario y su variación en las diferentes etapas del ciclo financiero.

En Anastasi y Balzarotti (2012), se analiza el funcionamiento de la incorporación de medidas anticíclicas dentro de un modelo teórico. En las hipótesis de comportamiento, se plantean una serie de ecuaciones en diferencia para determinar el nivel de créditos, el nivel de depósitos y las tasas de interés activas y pasivas. El modelo parte de una restricción presupuestaria dada por la hoja de balance de una entidad tipo, y capta la heterogeneidad de prestatarios a través de la segmentación entre diferentes clases de préstamos otorgados. Esta distinción permite incorporarle diferentes restricciones que se adecuan con las recomendaciones de capitales mínimos postuladas por BCBS en Basilea III (Bank for International Settlements, 2010), ya que separa los activos con y libres de riesgo. A su vez, se explican las modificaciones que se deberían aplicar sobre el modelo para considerar el capital Tier $1 \mathrm{y}$ el Tier $2 .{ }^{9}$

Otros modelos analizan la determinación del nivel de préstamos de equilibrio. Gaba (2012) realiza una introducción teórica excelente para

9 El capital Tier 1 (básico) se compone de un capital básico que consiste principalmente en las acciones ordinarias y las utilidades retenidas. En algunas ocasiones, puede incluir las acciones preferentes no-acumultivas. El capital Tier 2 (complementario) es mayor que el Tier 1, ya que incluye acciones preferentes con vencimiento fijo y deuda a largo plazo con un mínimo de vencimiento de más de cinco años. Este último queda subordinado a los depósitos y las afirmaciones del asegurador de depósitos. rea-lizar la construcción de esta clase de modelos. EI autor trabaja sobre una economía con un mercado financiero poco desarrollado, donde la determinación de la tasa de interés queda circunscripta al comportamiento del mercado bancario. A través del planteo de un modelo sencillo, en donde las variables endógenas corresponden al nivel de depósitos y préstamos, y las tasas de interés activas y pasivas, Gaba realiza un análisis de los elementos que componen el spread de tasas y también explica cómo una variación de esos componentes afecta el equilibrio existente. Del trabajo surge la siguiente conclusión: el mecanismo de trasmisión de política monetaria en países con mercados financieros poco profundos se produce a través del sistema bancario, sin ser necesaria la sustitución entre billetes y bonos descrita por los aportes keynesianos.

En Céspedes, Chang y Velasco (2012), se desarrolla un modelo donde interactúan el sector real y financiero de la economía. Una particularidad que presenta este modelo es que describe una economía pequeña y abierta, y, por lo tanto, da lugar a la discusión sobre el papel que juega el tipo de cambio en la determinación de los créditos otorgados por el sistema financiero. Además, incluye en el análisis medidas de políticas como son las restricciones en el mercado de divisas y el otorgamiento de créditos por parte del Gobierno. A partir de ese análisis, se ponen en evidencia los beneficios de la intermediación financiera. Si bien dentro del modelo se incorporan probabilidades de default e interacción con el lado real de la economía, no se distingue entre los diferentes acreedores y depositantes. Por este motivo, la discusión acerca de la evolución del riesgo sistémico dentro del sistema financiero queda de lado.

En Hahm et al. (2012) y Bruno y Shin (2013) se plantean modelos que resultan adecuados para analizar la evolución del riesgo sistémico del sector financiero. Estos modelos parten del análisis de la hoja de balance de una entidad financiera tipo, y además de diferenciar entre el capital propio y los pasivos, hacen una distinción entre los diferentes tipos de pasivos que conserva. Esto permite, por un lado, considerar la heterogeneidad de agentes, 
y diferenciar entre depositantes provenientes del lado real y del lado financiero de la economía. En estos modelos, una vez determinados los préstamos de equilibrio, se llega a la conclusión de que la distinción entre los diferentes tipos de pasivos es fundamental para poder explicar los problemas que existen actualmente en las recomendaciones realizadas en materia de regulación financiera.

Brunnermeier y Sannikov (2014) estudian la dinámica de equilibrio en una economía con fricciones financieras. Muestran que debido a los efectos de amplificación financiera, la economía es propensa a la inestabilidad y, de vez en cuando, entra en episodios de crisis. En el modelo, el riesgo, impulsado por la falta de liquidez de los activos, es incorporado como una variable endógena. A su vez, utilizan el coeficiente de apalancamiento para determinar la posibilidad de crisis. Muestran también cómo la creación de nuevos títulos de deuda puede dar lugar a un mayor apalancamiento y a crisis más frecuentes.

Una vez realizado este breve repaso de los modelos representativos del funcionamiento del sistema financiero, en el siguiente apartado se procede al armado de un modelo teórico matemático de este tipo. Los objetivos que persigue el planteo de este modelo son dos. En primer lugar, determinar el nivel de préstamos de equilibrio en una economía en la cual el sector financiero y el lado real de la economía interactúan. En segundo lugar, analizar la intervención estatal incorporando al Gobierno como tercer agente dentro del modelo planteado.
Se analizarán los impactos de una política en particular, a saber, un programa de créditos gubernamentales. Esos impactos serán de dos tipos, beneficiosos y perjudiciales. Por un lado, a través de un ejercicio matemático de estática comparada, se pondrán de manifiesto los beneficios de la intermediación financiera. Por el otro, quedará planteada la cuestión de lo discutido en la sección uno, acerca de la evolución del riesgo sistémico a partir de la profundización financiera y los perjuicios que conlleva.

Si bien en el armado del modelo no se utiliza una matemática compleja, permite una descripción de ciertas características inherentes al funcionamiento del sistema financiero. En particular, se podrá analizar el efecto del apalancamiento financiero, y cómo este mecanismo contribuye a profundizar en las medidas políticas implementadas. Sin embargo, la profundización del sistema financiero conlleva la gestación de un riesgo de carácter sistémico que puede llegar a afectar al producto real de la economía. Así, una medida política con el fin de aumentar el producto económico puede verse diezmada por la propia naturaleza del sector financiero.

\section{Modelo de dos agentes}

En este apartado, se plantea un modelo de dos periodos que analiza el comportamiento del sistema financiero dentro de una economía cerrada. En este modelo, se consideran dos tipos de agente.

Esquema del modelo

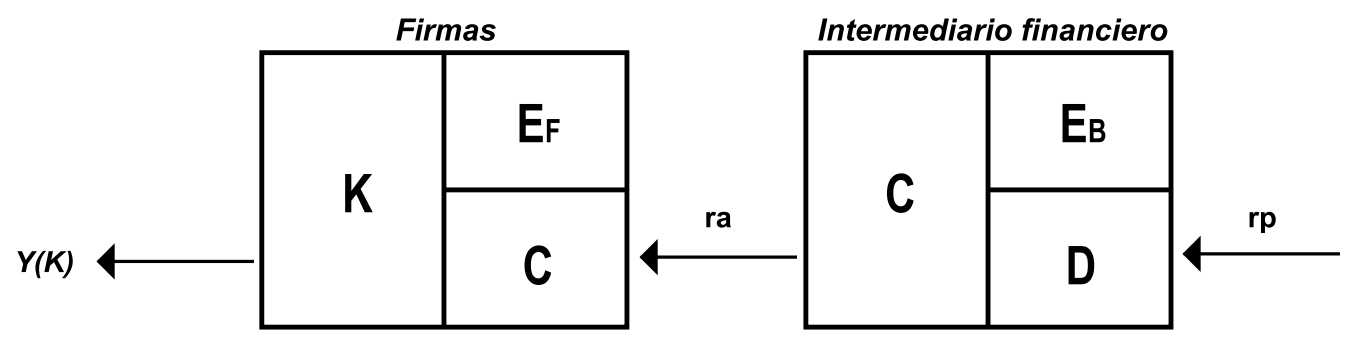

Fuente: elaboración de los autores. 
Uno intermediario financiero y firmas que operan en el lado real de la economía. El intermediario financiero realiza la oferta de préstamos para las firmas, las cuales demandan los préstamos que son destinados a aumentar el nivel de capital y, consecuentemente, el producto de esta economía. El equilibrio del modelo está determinado en el momento cero por la igualdad entre la oferta y la demanda de préstamos. Las variables endógenas estarán determinadas por el nivel de préstamos y la tasa de interés a la cual se otorgarán (figura 4). ${ }^{10}$

El intermediario financiero es un banco tipo. En su hoja de balance, por el lado de los activos, cuenta con los préstamos otorgados que son todos iguales entre sí. Por el lado de los pasivos, cuenta con capital propio y depósitos realizados por terceros. En el momento cero, el banco decide los préstamos a realizar y recibe depósitos de terceros como contrapartida. En el momento uno, recibe el pago del principal más los intereses de los préstamos otorgados y paga la deuda contraída más sus intereses. De acuerdo con lo detallado, el balance (la restricción presupuestaria) del banco tipo al momento cero puede escribirse como:

$C=D+E_{B}$

Donde $C$ indica la oferta de préstamos otorgada por el banco, $D$ los depósitos realizados por terceros y $E_{B}$ el capital bancario propio. Este último elemento del balance presupuestario se supondrá fijo en ambos periodos, a menos que sea alterado por un shock exógeno. Al ser un banco típico, la oferta de préstamos realizada depende positivamente de los intereses que cobra por estos; es decir, la tasa de interés activa $r_{a}$. A su vez, paga por los depósitos de terceros una tasa de interés pasiva $r_{p}$. Así, un cociente que indique el spread de tasas ${ }^{11}$ del banco tipo se encontrará dado por:

10 En este modelo, la tasa de interés endógena es la activa, dejando a la pasiva como variable exógena. Un trabajo donde esta última variable se hace endógena es en Bruno y Shin (2013), en el cual los depósitos de un intermediario financiero provienen de un banco mundial modelo.

11 En términos absolutos, el spread de tasas viene dado por la diferencia entre la tasa activa y la pasiva $s=r-r$ suponiendo que la diferencia entre ambas tasas es lo suficientemente $s=1+$ spread $=\frac{\left(1+r_{a}\right)}{\left(1+r_{p}\right)}$

En el momento uno, el banco cobra el principal más los intereses por los préstamos otorgados. De esta manera, los activos nocionales del banco vienen dados por $\left(1+r_{a}\right) L$. En ese mismo periodo, el banco paga los depósitos recibidos más los intereses, lo que conforma los pasivos nocionales del banco dados por $\left(1+r_{p}\right) D$. La función de beneficios del intermediario financiero quedará determinada por:

$\Pi=\left(1+r_{a}\right) C-\left(1+r_{p}\right) D$

El objetivo de este agente será maximizar su beneficio sujeto a una restricción colateral, la cual indica que las ganancias potenciales obtenidas en el momento uno deben superar los ingresos teniendo un posible default sobre los préstamos otorgados. Esto es:

$\left(1+r_{a}\right) C-\left(1+r_{p}\right) D>(1-a)\left(1+r_{a}\right) C$

Donde $a$ representa la probabilidad de default sobre los préstamos otorgados por el banco. Además, en el momento uno, este parámetro representa un coeficiente de apalancamiento estandarizado ${ }^{12}$ del intermediario financiero:

$a=\frac{\left(1+r_{p}\right) D}{\left(1+r_{a}\right) C}$

pequeña para que su producto con la tasa de interés pasiva tienda a cero, se puede ver que:

$1+s=\frac{\left(1+r_{a}\right)}{\left(1+r_{p}\right)} \Rightarrow r_{a}=s+r_{p}+s r_{p} \Rightarrow s=r_{a}-r_{p}$

El spread refleja la incidencia de ciertos componentes como la tasa de efectivo mínimo legal, los costos operacionales y la tasa de ganancia de los bancos y el riesgo de descalce (Gaba, 2012). La incidencia de estos componentes sobre el spread queda fuera del alcance de este artículo.

12 Definido el apalancamiento como el cociente entre activos sobre capital propio, este valor quedará comprendido entre cero e infinito, tendiendo a infinito a mayor nivel de apalancamiento. Otra manera de expresar este coeficiente es realizando el cociente entre el nivel de deuda sobre activos. Este valor quedará comprendido entre cero y uno, tendiendo a uno a medida que la entidad se encuentre más apalancada. 
Como se puede apreciar, $a$ se encuentra entre cero y uno, acercándose a uno mientras mayor sea el nivel de apalancamiento del banco. Cuando la restricción de colateral se encuentra activa (se cumple con igualdad) y se incorpora dentro de la misma restricción presupuestaria del banco tipo, se obtiene la función de oferta de préstamos bancarios. $^{13}$

$C_{s}=\frac{E_{B}}{1-s a}$

Reordenando la expresión de la oferta de préstamos, llamando $R=1+r_{p^{\prime}}$ y despejando para la tasa de interés activa, se obtiene la siguiente expresión:

$r_{a}=\left(\frac{R}{a}-1\right)-\frac{E_{B} R}{C a}$

Si se grafica la tasa de interés en función de los préstamos, la expresión anterior presenta una asíntota vertical para los préstamos al nivel de la tasa de interés activa dada por:

$r_{a}=\frac{R}{a}-1$

Hay que considerar una restricción adicional para la oferta de préstamos bancarios, relacionada con los beneficios de este agente. Si se expresan los ingresos netos esperados que obtiene el banco tipo por una unidad de crédito otorgada como $\left[\left(1+r_{a}\right)(1-a)\right]$, la oferta de préstamos se anulará para el siguiente tramo de tasa de interés activa:

$r_{a}<\frac{a}{1-a}$

Así, el nivel de oferta de préstamos que comienza a depender positivamente de la tasa de interés viene dado por:

$C_{0}=\frac{E_{B}}{1-\frac{a}{(1-a) R}}$

13 En la expresión presentada para la oferta de préstamos, se puede ver que la misma es un múltiplo del capital bancario. De esta manera, el coeficiente de apalancamiento de esta entidad viene dado por: $\frac{1}{1-s a}$
La gráfica que representa la tasa de interés en función de los préstamos otorgados se expresa mediante la siguiente función partida:

$r_{a}=\left\{\begin{array}{c}\overline{1-a} \quad \text { para } C<C_{0} \\ \left(\frac{R}{a}-1\right)-\frac{E_{B} R}{C a} \quad \text { para } C>C_{0}\end{array}\right.$

Gráfica 1.

Oferta de préstamos del banco tipo

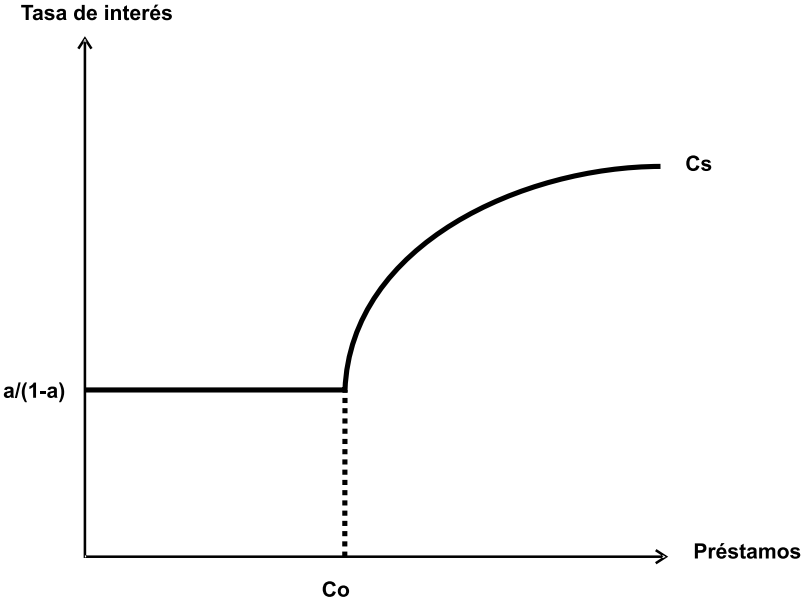

Fuente: elaboración de los autores con base en Céspedes et al. (2012).

Por el momento, solamente se ha explicitado la forma funcional de la oferta de préstamos otorgados por el banco tipo. Para determinar el equilibrio del modelo, hace falta encontrar la forma funcional de la demanda de préstamos. Como se ha supuesto en el modelo, la demanda de préstamos es realizada por las firmas que operan en el lado real de la economía, que presentan la siguiente función de producción:

$Y=A K^{a}$

Esta función utiliza como único insumo el capital que presenta rendimientos decrecientes de escala $(0<a<1)$. Este insumo se obtiene a partir del capital propio de las firmas y del nivel de préstamos que obtienen. De esta manera, la 
restricción presupuestaria de las firmas queda determinada por:

$K=C+E_{F}$

El capital será una función relacionada de manera inversa con la tasa de interés. En particular, se considerará como una renta perpetua que paga en cada periodo el monto $b$. Así, el capital se puede especificar mediante la siguiente forma funcional:

$K=b(r a)^{-1}$

La demanda de préstamos, realizada por las firmas que operan en el lado real de la economía, se deriva de la restricción presupuestaria. Al ser una demanda de préstamos tipo, depende negativamente de la tasa de interés que cobra el intermediario financiero. Esto es:

$C_{d}=b\left(r_{a}\right)^{-1}-E_{F}$

Manteniendo la consistencia con lo desarrollado hasta el momento, se debe expresar la tasa de interés en función de los préstamos. Esto es:

$r_{a}=\frac{b}{C+E_{f}}$

El equilibrio del modelo ${ }^{14}$ se encuentra determinado por la igualdad entre la oferta y la demanda de préstamos. Eso es:

$C_{s}=C_{d}$

14 Los valores de equilibrio en forma explícita, tanto para la tasa de interés como para los niveles de préstamos, quedan fuera del alcance de este artículo.
Gráficamente:

Gráfica 2.

Nivel de préstamos y tasa de interés de equilibrio

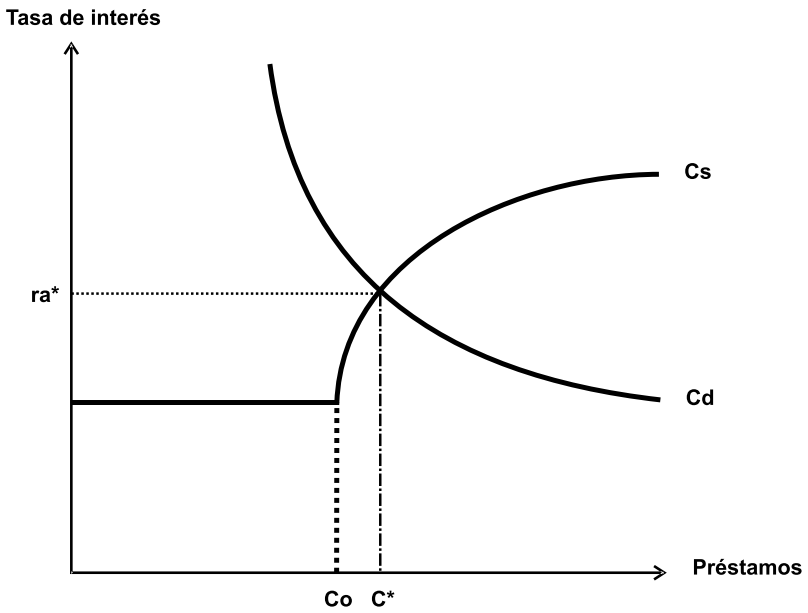

Fuente: elaboración de los autores con base en Céspedes et al. (2012).

\section{Modelo extendido a tres agentes: el rol del Gobierno}

En este apartado se incluye dentro del modelo al Gobierno como un tercer agente, el cual tiene bajo su control un banco con la capacidad de emitir dinero para otorgarlo en forma de subsidios, o en términos del modelo, en forma de préstamos adicionales. Este subsidio se puede otorgar directamente a las firmas para así financiar su producción, o al intermediario financiero para que este último aumente su capital bancario.

Este tercer agente se puede pensar como un banco que consigue financiamiento a una tasa muy baja (inferior a $r_{p}$ ) y que, además de encargarse de controlar la tasa de interés vía emisión monetaria, puede incidir sobre el producto a través de los subsidios que otorga. El financiamiento otorgado por el Gobierno, ya sea que esté dirigido a las firmas o al intermediario financiero, no tiene ningún costo.

El objetivo del ejercicio de estática comparativa que se presenta a continuación es mostrar cuál de los dos tipos de financiamiento posible 
que otorga el Gobierno a través de su propio banco tiene mayor impacto sobre el producto y la tasa de interés. Se considerará únicamente el caso en el que la restricción de colateral a la que se encuentra sujeto el banco tipo se halla activa; es decir, el tramo donde la demanda de préstamos intersecta a la oferta cuando esta última depende positivamente de la tasa de interés. Así se podrán apreciar las variaciones en la tasa de interés ante un cambio en el monto de préstamos de equilibrio.

En primer lugar, se considera el caso de un subsidio a las firmas. Dentro de este modelo, los subsidios se representan en forma de préstamos adicionales a los otorgados por el intermediario financiero. Esto es, el Gobierno, en el momento cero, aumenta la oferta de préstamos bancarios en un monto $L$. Consecuentemente, el nuevo equilibrio del mercado de préstamos queda determinado por:

$C_{d}=C_{s}+L=C_{s}^{\prime}$

Esto implica un desplazamiento horizontal de la oferta de préstamos. Como se supuso que la restricción de colateral permanecía activa, el resultado del otorgamiento de subsidios a las firmas deriva en un incremento en el nivel de préstamos otorgados y una reducción en la tasa de interés de equilibrio, lo que explica la mayor demanda de créditos.

Matemáticamente, cuando la restricción de colateral se encuentra activa (lo que se supuso al inicio de este ejercicio), la nueva función de oferta de préstamos, en esta economía, queda determinada por:

$C_{s}^{\prime}=\frac{E_{B}}{1-s a}+L$

Donde el primer término representa la oferta de préstamos bancarios y el segundo, el otorgamiento de subsidios por parte del Gobierno. Despejando la tasa de interés activa, y recordando que $R=1+r_{p}$, se obtiene la siguiente expresión:

$r_{a}=\left(\frac{R}{a}-1\right)-\frac{E_{B} R}{(C+L) a}$
Así, el nivel de oferta de préstamos que comienza a depender positivamente de la tasa de interés ahora viene dado por:

$C_{0}{ }^{\prime}=\frac{E_{B}}{1-\frac{a}{(1-a) R}}+L=C_{0}+L$

La gráfica que representa la tasa de interés en función de los préstamos otorgados se expresa mediante la siguiente función partida:

$r_{a}=\left\{\begin{array}{c}\frac{a}{1-a} \quad \text { para } C<C_{0}{ }^{\prime} \\ \left(\frac{R}{a}-1\right)-\frac{E_{B} R}{(C+L) a} \quad \text { para } C>C_{0}\end{array}\right.$.

Gráficamente, esta variación se representa con un desplazamiento hacia la derecha de la oferta de préstamos de esta economía. En particular, es un desplazamiento del monto $C_{o}$ en una cuantía de $L$ (gráfica 3).

Gráfica 3.

Subsidio gubernamental a las firmas

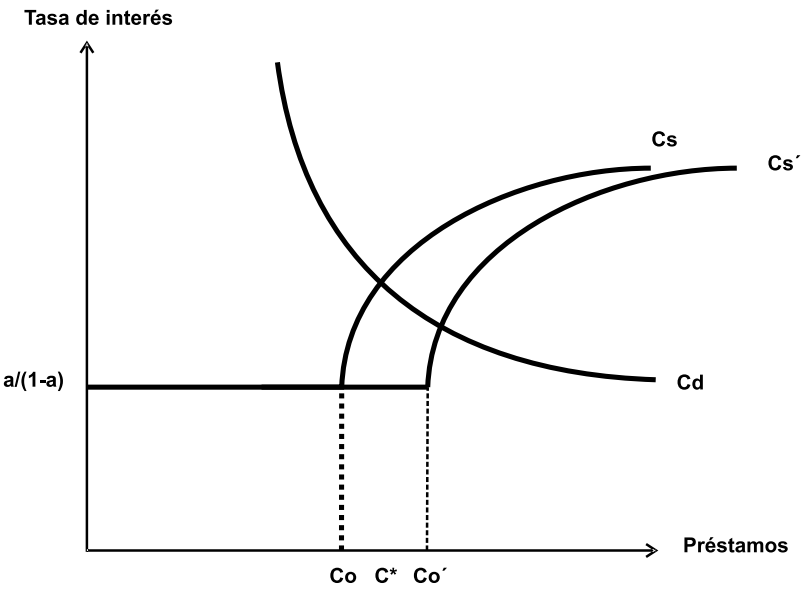

Fuente: elaboración de los autores con base en Céspedes et al. (2012).

El resultado final de la política gubernamental efectuada puede sintetizarse de la siguiente manera. Un aumento del nivel de préstamos ofrecidos 
dentro de esta economía conduce a una disminución de la tasa de interés y, consecuentemente, a un aumento del nivel del producto. Sin embargo, hay que tener en cuenta que las firmas demandan crédito por una cantidad igual a la diferencia entre su demanda de capital y el valor de su patrimonio neto, cantidad que no ha sido modificada por la política gubernamental que se está analizando. De esta manera, si la restricción del mercado financiero se encuentra activa, los bancos otorgan un nivel de crédito cada vez menor a medida que la tasa de interés activa va disminuyendo a causa de un aumento de los subsidios. Esto se explica por una disminución de su coeficiente de apalancamiento que depende positivamente de la tasa de interés activa.

Supóngase ahora que el Gobierno, en lugar de financiar a las firmas a través de un otorgamiento de préstamos adicionales, otorga un monto $L$ al intermediario financiero. Este financiamiento se incorpora dentro de la hoja de balance del banco tipo, por lo que aumenta el capital propio $y$, consecuentemente, se modifica la oferta de préstamos bancarios. Matemáticamente, cuando la restricción de colateral se encuentra activa (lo que se supuso al inicio de este ejercicio), la nueva función de oferta de préstamos en esta economía queda determinada por:

$C_{S}{ }^{\prime \prime}=\frac{E_{B}+L}{1-s a}$

Despejando para la tasa de interés activa, y recordando que $R=1+r_{p}$, se obtiene la siguiente expresión:

$r_{a}=\left(\frac{R}{a}-1\right)-\frac{\left(E_{B}+L\right) R}{C a}$

Así, el nuevo nivel de oferta de préstamos, que comienza a depender positivamente de la tasa de interés, ahora viene dado por:

$C_{0}^{\prime \prime}=\frac{E_{B}+L}{1-\frac{a}{(1-a) R}}$
Sabiendo que $0<1-\frac{a}{(1-a)_{R}}<1$, se puede afirmar que $C_{o}{ }^{\prime \prime}>C_{o}$ '. La gráfica que representa la tasa de interés en función de los préstamos otorgados se expresa mediante la siguiente función partida:

$r_{a}=\left\{\begin{array}{c}\frac{a}{1-a} \quad \text { para } C<C_{0}{ }^{\prime \prime} \\ \left(\frac{R}{a}-1\right)-\frac{\left(E_{B}+L\right) R}{C a} \quad \text { para } C>C_{0}{ }^{\prime \prime}\end{array}\right.$

Gráficamente, esta variación se representa con un desplazamiento hacia la derecha de la oferta de préstamos de esta economía (gráfica 4). En particular, es un desplazamiento del monto $C_{o}$

en una cuantía de $\left(1-\frac{a}{(1-a) R}\right)^{-1}$.

Gráfica 4.

Subsidio gubernamental al intermediario financiero

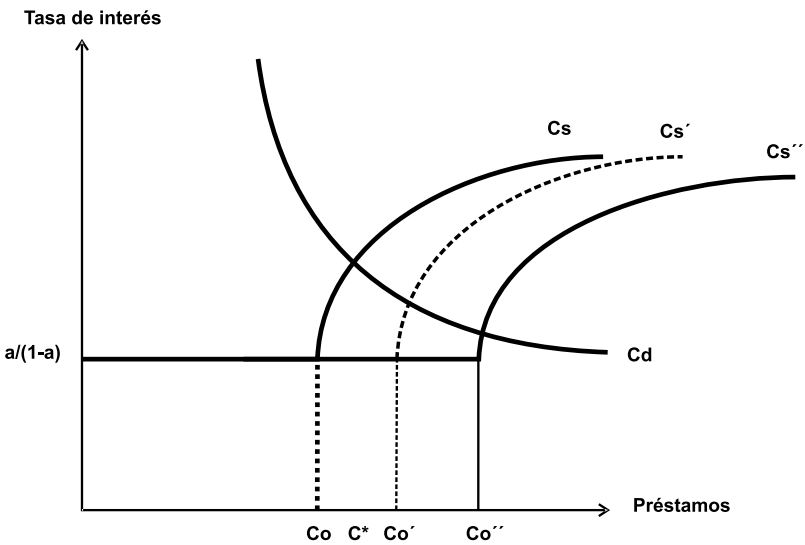

Fuente: elaboración propia con base en Céspedes et al. (2012).

El nuevo equilibrio estaría determinado por:

$C_{d}=C_{s}^{\prime \prime}$

Como se supuso que la restricción de colateral permanecía activa, el resultado del otorgamiento de subsidios a la entidad financiera es un incremento en el nivel de préstamos otorgados y una reducción en la tasa de interés de equilibrio. 
A diferencia del subsidio a las firmas, el desplazamiento de la curva de oferta de préstamos es mayor. Ahora, el nivel de préstamos que comienza a depender positivamente de la tasa de interés no aumenta en $L$, sino que lo hace en $L$ veces multiplicado por el coeficiente $\left(1-\frac{a}{(1-a)_{R}}\right)^{-1}$, expresión donde se encuentra involucrado el coeficiente de apalancamiento del banco tipo.

Como el coeficiente de apalancamiento es mayor a uno, el desplazamiento de la curva de oferta de préstamos es mayor cuando se subsidia al intermediario financiero que cuando se otorga un subsidio directo a las firmas. Consecuentemente, se concluye que utilizando el canal de la intermediación financiera es posible alcanzar un mayor nivel de la cantidad de préstamos de equilibrio y una tasa de interés menor. Así, el producto de la economía obtenido por las firmas también es mayor. De esta manera, se corrobora la hipótesis de que un programa de créditos impulsado por el Gobierno es más efectivo, en términos de aumento del producto, si se realiza a través de un intermediario financiero que si se otorga un subsidio directo a las firmas que operan en el lado real de la economía.

Hasta el momento, solo se han analizado los beneficios que conlleva la intermediación financiera; sin embargo, queda pendiente el desarrollo de la cuestión planteada en la sección uno, referente a la gestación del riesgo sistémico. Para llevar a cabo esta explicación, los conceptos de prociclicidad y apalancamiento son introducidos nuevamente. Este último fue desarrollado dentro del modelo matemático, y es clave en la comprensión de los beneficios de contar con un intermediario bancario. Sin embargo, la prociclicidad no es independiente de la evolución temporal del producto $y$, al tratarse de un modelo en el que se determina un equilibrio estático, es un concepto que ha sido dejado de lado. Por este motivo, el análisis de la gestación del riesgo sistémico se desarrollará fuera del modelo; se especificarán las consecuencias que podría llegar a tener un aumento de capital bancario propio.

Como se ha analizado dentro del modelo, el otorgamiento de subsidios por parte del Gobierno es más efectivo si se realiza a través de un intermediario financiero. Esto radica en el mecanismo de apalancamiento implementado por el banco tipo. Sin embargo, además de un mayor aumento en el nivel de producto, el aumento del capital bancario genera consecuencias en lo que respecta a profundización financiera.

Siguiendo la lógica desarrollada en la explicación del concepto de apalancamiento, se puede argumentar que ante el otorgamiento de subsidios gubernamentales al intermediario financiero, el coeficiente de apalancamiento de este último disminuye, ya que el mayor nivel de préstamos otorgados por esta entidad está respaldado en su totalidad por el incremento del capital propio. Consecuentemente, suponiendo que la regulación financiera vigente en esta economía exigiese un ratio de capital constante, ${ }^{15}$ la entidad financiera reacomodará su estructura dentro del balance para poder alcanzar el nivel de apalancamiento original y así aumentar sus ganancias. Para conseguir su cometido, la entidad incrementará su nivel de deuda, lo que, a su vez, aumentará aún más los préstamos otorgados, hasta conseguir recomponer plenamente su coeficiente de apalancamiento. Suponemos que este proceso finaliza cuando la entidad alcanza el nivel mínimo de capital propio exigido por los reguladores.

Este proceso de reacomodación en la estructura del balance bancario, en pos de un aumento de los beneficios del intermediario financiero, no está exento de la generación de perjuicios en el funcionamiento futuro de la economía. El inconveniente surge en el momento en que el aumento de la hoja de balance del sector bancario se produce de manera exógena por un subsidio gubernamental. En el proceso de recomposición del coeficiente

15 Desde la década de los ochenta (década en la que se lanzaron las primeras recomendaciones realizadas por el BCBS bajo el nombre de Basilea I), la exigencia de capitales mínimos fue el principal requisito de la regulación financiera. Con el pasar del tiempo y la evolución de las crisis financieras, estos ratios se fueron endureciendo y ajustando en Basilea II (Bank for International Settlements, 2006) y Basilea III (Bank for International Settlements, 2010). Una buena referencia de la evolución de los requisitos de capitales mínimos puede obtenerse en Carmassi y Micossi (2012). 
de apalancamiento, el intermediario financiero sale en búsqueda de una mayor cantidad de depósitos. Ese tipo de deuda generalmente proviene de otras entidades financieras, y es a una tasa alta y a un plazo corto. Este mecanismo termina profundizando las interconexiones dentro del sistema financiero, y gesta así un potencial riesgo sistémico que, en el caso de materializarse, anula por completo los objetivos perseguidos por la implementación de la política gubernamental de otorgamiento de subsidios.

\section{CONCLUSIÓN}

El artículo se ha estructurado de la siguiente manera: luego de la introducción, en la primera sección se desarrollan los conceptos de prociclicidad, apalancamiento y riesgo sistémico, los cuales son característicos del sistema financiero. En el primer apartado, se explica la prociclicidad, concepto que está directamente ligado a la evolución del ciclo real, haciendo que las fluctuaciones del ciclo financiero sean más pronunciadas que las fluctuaciones del producto. También, dentro del primer apartado, se mencionaron tres fuentes que generan que el negocio bancario sea procíclico; la primera está referida a aspectos propios de la actividad financiera; la segunda, a las fallas de mercado existentes dentro del sector, y la última, a las propias regulaciones financieras.

En el siguiente apartado, se define el concepto de apalancamiento bancario a partir de la hoja de balance del sistema financiero, que está vinculado directamente con el primer concepto desarrollado (prociclicidad); se explica por qué presenta la característica de ser procíclico. Seguidamente, mediante un ejemplo sencillo, se muestra que, a pesar de los intentos regulatorios de exigir un apalancamiento constante, la reacomodación de los pasivos que realizan las entidades financieras en la etapa de crecimiento económico hace que el sector sea altamente inestable; esta inestabilidad se ve aún más pronunciada en presencia de un apalancamiento procíclico.

En el último apartado, a partir de las definiciones de prociclicidad y apalancamiento, se define el concepto de riesgo sistémico. Luego de una descripción de fenómenos que caracterizan este tipo de riesgo, al indagar sobre la variación en la composición de los pasivos de las hojas de balance del sector financiero en diferentes etapas del ciclo económico, se establece una relación entre los tres conceptos desarrollados.

En la segunda sección, se fundamenta la metodología de análisis de este artículo, que consiste en el planteo de un modelo teórico matemático de determinación de préstamos y tasa de interés de equilibrio. Esta sección comienza con una revisión de los modelos representativos del sistema financiero. Posteriormente, se establecen las hipótesis de comportamiento del modelo, donde se incorporaron los elementos necesarios para analizar los conceptos definidos en la primera sección. Finalmente, se propone un modelo teórico simple que permite entender la interacción y articulación entre el sector público y el sector financiero. Sobre el mismo se realiza el ejercicio de asumir un shock exógeno dado por un programa gubernamental de créditos.

El aporte de este artículo, más allá de evaluar la relación entre el tamaño del sistema financiero y el crecimiento del producto (Beck, Degryse \& Kneer, 2014; Chen \& Imam, 2014), es el armado de un modelo teórico matemático que representa adecuadamente la naturaleza de la intermediación financiera, y que permite dilucidar los beneficios y los perjuicios de los programas de créditos gubernamentales dirigidos a la economía real a través del sector financiero. Formalmente, luego de plantear un modelo de dos agentes -intermediario financiero y firmas-, se incorpora un tercer agente, el Gobierno, y se demuestra que el otorgamiento de subsidios por parte de este es más efectivo si se realiza a través de un intermediario financiero a causa del mecanismo de apalancamiento implementado por el banco tipo.

Sin embargo, además de un mayor aumento en el nivel de producto, el aumento del capital bancario, proceso de reacomodación de pasivos mediante, termina profundizando las interconexiones dentro del sistema financiero y gestando, así, 
un potencial riesgo sistémico que en el caso de materializarse, anularía por completo los objetivos perseguidos por la implementación de la política gubernamental de otorgamiento de subsidios.

Por las razones desarrolladas a lo largo del artículo, se considera que la regulación del sistema financiero es de suma importancia. Sin embargo, la regulación financiera vigente, y como esta ha ido avanzando en los últimos años en materia de política económica, está lejos de poder prevenir o predecir las crisis generadas dentro de este sector, generalmente de carácter sistémico. Las políticas económicas que son de carácter vinculante con los intermediarios financieros deben ser sumamente cautelosas y considerar los efectos adversos que pueden llegar a generar sobre la economía real. Tener en cuenta los elementos característicos del sector financiero que contribuyen con la gestación de riesgos sistémicos, sería un buen punto de partida para los hacedores de política económica.

\section{REFERENCIAS}

1. Adrian, T. \& Shin, H. S. (2013). Procyclical leverage and value-at-risk. Federal Reserve Bank of New York. Staff Reports, 338.

2. Anastasi, A. \& Balzarotti, V. (2012). ¿Colchones contables o de liquidez? Los riesgos de subestimar aspectos financieros de las reglas anticíclicas. Banco Central de la República Argentina. Ensayos económicos, $67,73-120$.

3. Bank for International Settlements (BIS). (2006). Convergencia internacional de medidas y normas de capital. Suiza: BIS.

4. Bank for International Settlements (BIS). (2010). Basilea III: marco regulador global para reforzar los bancos y sistemas bancarios. Suiza: BIS.

5. Bebczuk, R. (2003). Asymmetric information in financial markets: introduction and applications. United Kingdom: Cambridge University Press.

6. Beck, T., Degryse, H. \& Kneer, C. (2014). Is more finance better? Disentangling intermediation and size effects of financial systems. Journal of Financial Stability, 10, 50-64.

7. Borio, C. (2012). The financial cycle and macroeconomics: What have we learned? BIS Working Paper, 395.

8. Brunnermeier, M. K. \& Sannikov, Y. (2014). A macroeconomic model with a financial sector. The American Economic Review, 104(2), 379-421.

9. Bruno, V. \& Shin, H. (2013). Capital flows, cross-border banking and global liquidity. National Bureau of Economic Research. NBER Working Paper, 19038.

10. Carmassi, J. \& Micossi, S. (2012). Time to set banking regulation right. Financial Markets Group Research Centre. Brussels: Centre for European Policy Studies (CEPS).

11. Céspedes, L. F., Chang, R. \& Velasco, A. (2012). Financial intermediation, exchange rates, and unconventional policy in an open economy. National Bureau of Economic Research, w18431.

12. Chen, J. \& Imam, P. (2014). Consequences of asset shortages in emerging markets. Macroeconomics and Finance in Emerging Market Economies, 7(1), 4-35.

13. De Bandt y Hartmann. (2000). Systemic risk: A survey. ECB. Working Paper, 35.

14. Gaba, E. (2012). Teoría y práctica sobre tasa de interés de equilibrio. Facultad de Ciencias Económicas (Working paper). Maestría en Gestión Económica y Financiera de Riesgos, Facultad de Ciencias Económicas, Universidad de Buenos Aires. 
15. Goodhart, C. A., Sunirand, P. \& Tsomocos, D. P. (2006). A model to analyse financial fragility. Economic Theory, 27(1), 107-142.

16. Gual, J. (2009). El carácter procíclico del sistema financiero. Documento de economía, 14.

17. Hahm, J. H., Shin, H. S. \& Shin, K. (2012). Non-core bank liabilities and financial vulnerability. National Bureau of Economic Research. NBER Working Paper, 18428.

18. Hamann, F., Hernández, R., Silva, L. \& Tenjo, F. (2013). Credit pro-cyclicality and bank balance sheet in Colombia. Colombia: Banco de la República.

19. Marcheggiano, G., Miles, D. \& Yang, J. (2013). Optimal bank capital. The Economic Journal, 123(567), $1-37$.

20. Mishkin, F. (2007). The economics of money, banking, and financial markets. London: Pearson Education.

21. Powell, A., Miller, M. \& Maier, A. (2011). Prudent banks and creative mimics: Can we tell the difference? Centre for Competitive Advantage in the Global Economy. Working Paper, 76. 